\title{
Quasi-free neutron and proton knockout reactions on ${ }^{57} \mathrm{Ni}$
}

\section{Alina Movsesyan ${ }^{* 1,2} ;$ Stefanos Paschalis ${ }^{1,3}$, Thomas Aumann ${ }^{1,2}$, Dominic Rossi ${ }^{2,4}$ for the $\mathbf{R}^{3} \mathbf{B}$-collaboration}

${ }^{1}$ Institut für Kernphysik, Technische Universität Darmstadt, D-64289 Darmstadt, Germany

${ }^{2}$ GSI Helmholtzzentrum für Schwerionenforschung GmbH, D-64291 Darmstadt, Germany

${ }^{3}$ University of Liverpool, Liverpool L69 7ZE, United Kingdom

${ }^{4}$ Institut für Kernchemie, Johannes Gutenberg-Universität, D-55128 Mainz, Germany

This work presents one of the several experiments at the $\mathrm{R}^{3} \mathrm{~B}$-LAND setup (at GSI in Darmstadt, Germany), dedicated to investigations of nuclear structure with proton-induced quasi-free scattering reactions in inverse kinematics at relativistic energies. Kinematically complete measurements were undertaken, which allow for the analysis of $(p, 2 p)$ and (p,pn) reactions. A similar technique with an upgraded setup will be used for the future $\mathrm{R}^{3} \mathrm{~B}$ program at FAIR/GSI, for studies of the single-particle structure and the role of $\mathrm{N}-\mathrm{N}$ correlations in very exotic nuclei. One-neutron (p,pn) and one-proton (p,2p) quasi-free knockout reactions on the ${ }^{57} \mathrm{Ni}$ nucleus are being analyzed within this work. Position, time-of-flight and energy-loss measurements of heavy ions and light particles before and after the target were undertaken. A clean identification of the quasi-free scattering reactions on a proton target can be achieved with the detector system around the target area, in combination with the exclusive identification of the reaction participants.

52 International Winter Meeting on Nuclear Physics - Bormio 2014,

27-31 January 2014

Bormio, Italy

\footnotetext{
* Speaker.

†E-mail: a.movsesyan@gsi.de
} 


\section{Introduction}

Within the last century, a tremendous effort was put into the developing new experimental techniques and theoretical models for nuclear science, in order to explore and explain the nuclear structure and properties, not only of the stable nuclei, but also of those with a high neutron-proton asymmetry, which are closer to the drip-lines. The main interest behind the presented work is the study of single-particle (SP) structure of nuclei far from the valley of beta-stability and the tools best suited for the dedicated studies. In order to explore the SP properties of nuclei, direct reactions have been widely used, which proceed without a formation of a compound nucleus and result only in a transfer or removal of one (or a few) nucleons from the nucleus of interest. They have proved to be very powerful in probing the spectroscopic strength of the SP levels both in stable and exotic nuclei [1].

The first consistent quantitative body of evidence of reduced spectroscopic strength was reported for stable nuclei from $\left(\mathrm{e}, \mathrm{e}^{\prime} \mathrm{p}\right)$ reaction measurements within a wide mass range of $\mathrm{A}=7$ 208 [2]. Long-range correlations (LRC) and short-range correlations (SRC) have been shown to contribute to this reduction. Illustrative examples of the extent to which the SRC and LRC influence the SP properties in the nuclear interior and closer to the Fermi surface can be found in Refs. [3, 4]. There has been a significant experimental and theoretical effort in the last decades to measure and describe the strengths of these correlations, their isospin dependence and the effects on the single particle properties (e.g. [2, 5, 6, 7, 8] and Refs. therein).

Despite being much easier to handle theoretically and providing spectroscopic information with a good precision, the $\left(\mathrm{e}, \mathrm{e}^{\prime} \mathrm{p}\right)$ reactions are limited to stable nuclei and proton probes. In contrary, one-nucleon removal (particularly, hadronic induced knockout) reactions in combination with inverse kinematics give a possibility to probe both the proton and the neutron single-particle levels. An intriguing systematical analysis of the SP strength reduction was presented in Ref. [9], based on several experimental data from proton and neutron knockout reactions induced on composite targets (e.g. Be). A comparison with $\left(\mathrm{e}, \mathrm{e}^{\prime} \mathrm{p}\right)$ results revealed a good agreement of the observed nearly constant reduction of spectroscopic strength to about $60-65 \%$ in the region around the stable nuclei. However, for exotic nuclei with a large neutron-proton asymmetry, data from one-neutron and one-proton knockout reactions reveal a dependency on the difference of proton-neutron Fermi levels: for the loosely bound excessive species there is almost no or only a very weak reduction observed, whereas a strong quenching is observed for the more deficient, strongly bound nucleons (see Fig.6 in Ref. [9]). There are works ([10],[11]) predicting correlation effects in very asymmetric nuclei that would result in a similar behavior, but not as dramatic as presented in [9]. The origin of the strong quenching is still not understood. However, since the knockout reactions on light targets at intermediate energies are mostly peripheral and strongly model dependent, a validation of these findings is needed with tools more sensitive to the nuclear interior and less model dependent, e.g. quasi-free scattering (QFS) reactions.

\section{QFS at $\mathbf{R}^{3} \mathbf{B}$}

The quasi-free scattering or knockout reactions on proton targets in inverse kinematics comprise an important part of the research program of the $\mathrm{R}^{3} \mathrm{~B}$-collaboration (Reactions with Relativistic 
Radioactive Beams) [12], addressing the structure of exotic nuclei far from the valley of betastability, their single particle and collective properties. In terms of inverse kinematics, the quasi-free scattering reactions proceed via a single interaction between a high-energy nucleon in a projectile nucleus and the proton target. At projectile energies of a few hundred $\mathrm{MeV} /$ nucleon, this interaction is strongly localized and the process of knocking out a nucleon can be viewed as an interaction between a (quasi) free nucleon of the projectile nucleus and the proton. The proton target (e.g., liquid hydrogen, $\mathrm{CH}_{2}$, etc.) increases the sensitivity to the more deeply bound states. Additionally, if the incident energy is appropriately chosen, the rescattering in the final state can be minimized, since the nucleon-nucleon interaction cross-section reaches its minimum around $250-350 \mathrm{MeV}$. The inverse kinematics, combined with the measurements of time-of-flight, energy-loss and position in the incoming and outgoing channels, allows for an exclusive reconstruction of properties of all participants of the reactions, i.e. the projectile nucleus, the residual projectile-like fragment (for which a detection and calorimetry of prompt deexcitation gamma-rays is also available), the recoiled target proton and the knocked out nucleon. This is the concept of the $\mathrm{R}^{3} \mathrm{~B}$ setup, which is planned to be installed at a high-energy branch of the future FAIR facility [13] and which will benefit from the highly energetic and very exotic beams provided by the enhanced production and acceleration complex. The concept and the tools for the future program are being developed and tested at the present $\mathrm{R}^{3} \mathrm{~B}$-LAND setup at GSI.

\section{The ${ }^{57} \mathrm{Ni}$ isotope}

The current work addresses an experiment of quasi-free knockout reactions on $\mathrm{Ni}$ isotopes. The doubly magic (yet unstable) ${ }^{56} \mathrm{Ni}$ nucleus with $\mathrm{Z}=\mathrm{N}=28$ closed shells and the SP levels of nuclei around this shell gap have been addressed in several experimental $[14,15,16,17,18]$ and theoretical [19] works. In order to prove the applicability of the setup to investigations of SP properties of the exotic nuclei, a benchmark case of quasi-free one-nucleon knockout from ${ }^{57} \mathrm{Ni}$ will be discussed in this work, for which experimental and theoretical data are available. A large reduction of the SP strength for neutrons (compared to shell-model calculations) was observed in Ref. [17] via neutron knockout reaction from ${ }^{57} \mathrm{Ni}$ on a Be target. The authors report a reduction factor of $\mathrm{R}_{s}=0.55$ and $\mathrm{R}_{s}=0.47$ for neutron knockout to the ground- and to the excited states of ${ }^{56} \mathrm{Ni}$, respectively. In Ref. [19], an FRPA (Fadeev Random Phase Approximation Method) calculation of the spectroscopic factors, including SRC, LRC and configuration mixing, resulted in a spectroscopic factor of 0.62 for the $v 2 p_{3 / 2}$ shell (given as a fraction of Independent Particle Model (IPM)). This is comparable with the corresponding value from Ref. [17], recalculated to be 0.58(11) relative to IPM. In contrast to these works, authors of Ref. [18] find a small quenching in a study of angular distributions in the ${ }^{56} \mathrm{Ni}(\mathrm{d}, \mathrm{p})^{57} \mathrm{Ni}$ transfer reaction [16]. The inconsistency between spectroscopic factors from transfer and knockout reactions, which was also observed for other nuclei (see e.g. Refs. [20],[21]), is still an unsolved problem.

\section{Setup and experimental technique}

Due to the broad range of physics goals of the $\mathrm{R}^{3} \mathrm{~B}$-collaboration and the various reaction types employed in the studies, a versatile setup is needed, capable of high-resolution and high-efficiency 
measurements. Different units of such a detector system are nowadays in development, testing and production phases among the international member institutions of the collaboration. However, already throughout the last decades the concept of the future $\mathrm{R}^{3} \mathrm{~B}$ setup has been used in many experiments on several generations of the ALADIN/LAND setup at the high energy branch of the current GSI facility. The setup used in the presented experiment is shown in Fig. 1. It is equipped

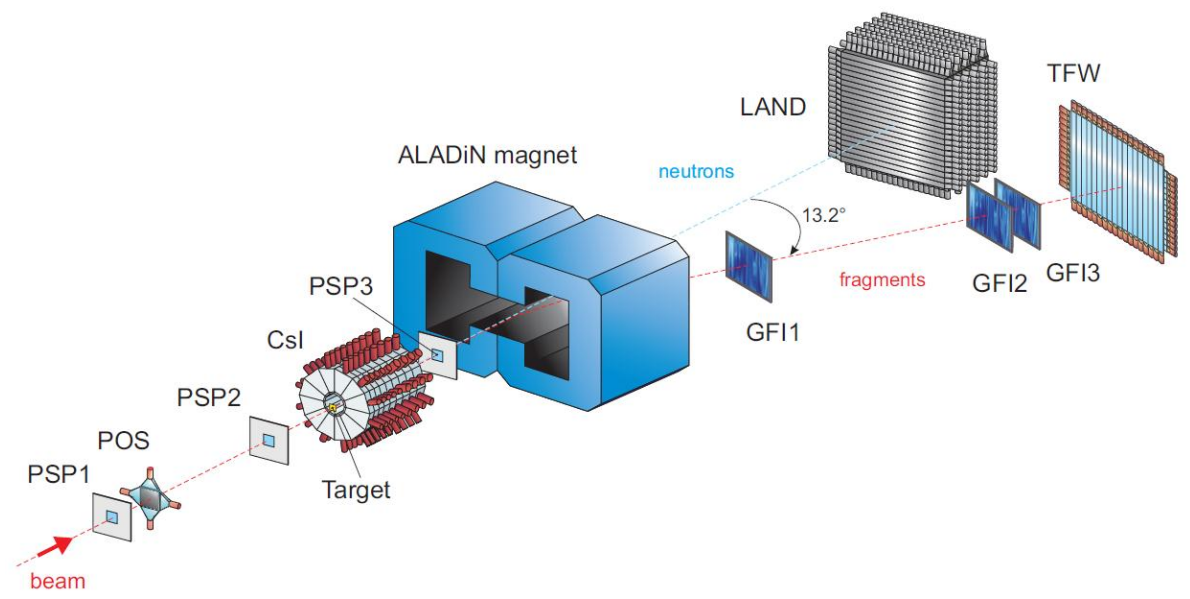

Figure 1: The $\mathrm{R}^{3} \mathrm{~B}-\mathrm{LAND}$ setup, used in the discussed experiment. Picture taken from Ref. [22].

with a detection system for the identification and tracking of the incoming isotopes impinging on a production target. The secondary cocktail beams were produced via projectile fragmentation of primary ${ }^{58} \mathrm{Ni}(600 \mathrm{AMeV})$ and ${ }^{78} \mathrm{Kr}(700 \mathrm{AMeV})$ beams on a Be $\left(4.19 \mathrm{~g} / \mathrm{cm}^{2}\right)$ target. The in-flight selection based on the so-called $\mathrm{B} \rho-\Delta \mathrm{E}-\mathrm{B} \rho$ method was performed at the FRS fragment separator [23]. The production target for the investigation of knockout reactions on protons was made of polyethylene $\left(\mathrm{CH}_{2}, 216 \pm 4 \mathrm{mg} / \mathrm{cm}^{2}\right)$. In order to estimate the contribution of the carbon content in $\mathrm{CH}_{2}$ and the background of the setup, data with a carbon target (C, $\left.187 \pm 4 \mathrm{mg} / \mathrm{cm}^{2}\right)$ and without a target were taken as well. The projectile-like reaction products are deflected in the large acceptance dipole magnet ALADIN towards the fragment branch of the setup. The LAND neutron detector [24] was not used for the current analysis due to the reaction kinematics. The recoiled protons, knocked out light particles and prompt $\gamma$-rays are detected with a dedicated detection system around the target. It comprises a $2 \pi$ highly segmented barrel of $144 \mathrm{CsI}(\mathrm{Na})$ crystals ( $\gamma$-rays, $\mathrm{p}$, n) and a plastic detector with 12 paddles, used as a proton trigger. An event-by-event analysis of energy-loss, time-of-flight and position measurements before and after the target, combined withthe $\gamma$-coincidence analysis, allows for kinematically complete measurements of the incoming and outgoing channels.

\section{5. ${ }^{57} \mathrm{Ni}(\mathbf{p}, \mathbf{p n}){ }^{56} \mathrm{Ni}$ and ${ }^{57} \mathrm{Ni}(\mathbf{p}, 2 \mathbf{p}){ }^{56} \mathrm{Co}$ reactions}

The identification of the incoming cocktail beam is done via velocity, energy-loss and magnetic rigidity analysis and is presented in a $2 \mathrm{D}$ plot of mass-over-charge ratio against the reconstructed 
charge in Fig. 2(a). The charge of the outgoing heavy fragments is analysed twice - right after the target and at the end of the setup (see Fig. 2(b)). Thus, the fragments that undergo a breakup

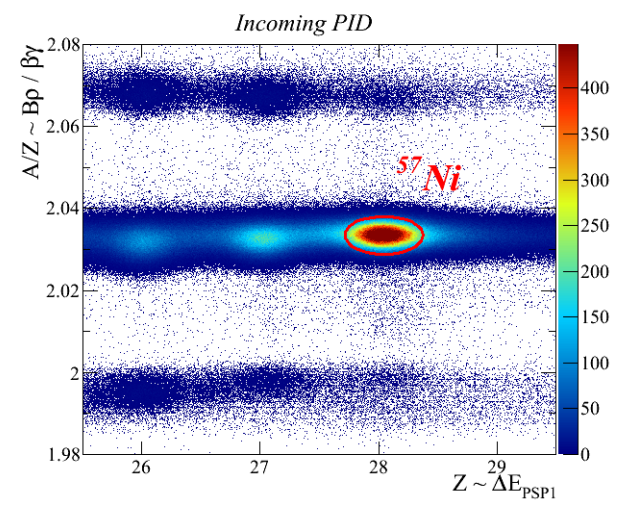

(a) Incoming beam ID

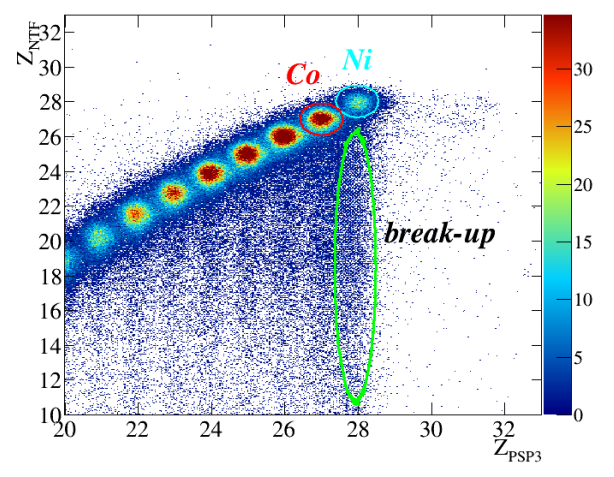

(b) Outgoing charge ID

Figure 2: a) Identification of the incoming cocktail beam by means of mass-over-charge $(A / Z)$ and charge $(Z)$ reconstruction and the selection of ${ }^{57} \mathrm{Ni}$ ions (red ellipse). b) Correlation of reconstructed charges. The $\mathrm{Ni}$ and Co fragments are selected with blue and red ellipses, respectively. The tail indicated by the green ellipse is formed as a result of fragment breakup before reaching the detector at the end of the setup.
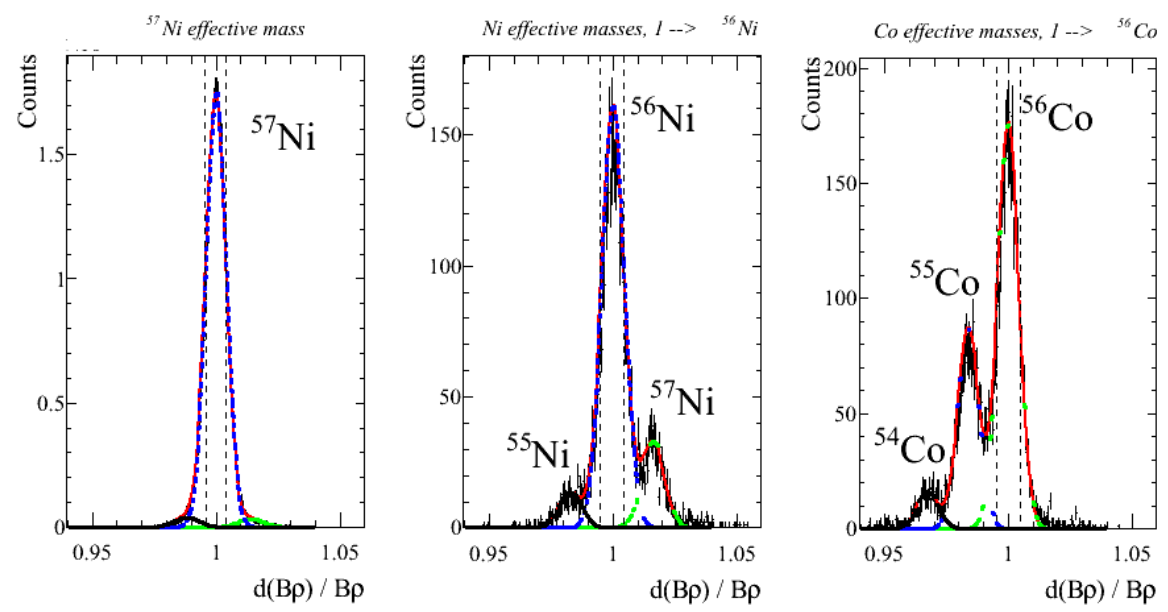

Figure 3: Effective mass distributions expressed by means of the relative rigidity for $\mathrm{CH}_{2}$ target data. The left panel shows the unreacted ${ }^{57} \mathrm{Ni}$ isotopes. The mass distributions in the middle and the right panels are obtained with the requirements of the incoming ${ }^{57} \mathrm{Ni}$ and outgoing $\mathrm{Ni}(\mathrm{Z}=28)$ and $\mathrm{Co}(\mathrm{Z}=27)$ nuclei.

(green ellipse) are eliminated from the analysis. The masses of the selected outgoing fragments are reconstructed based on position measurements before and after ALADIN and by using a dedicated tracking procedure. The identification of the unreacted (left) and projectile-like reaction fragments (middle, right) is shown in Fig. 3, by means of the magnetic rigidity analysis relative to a reference trajectory.

The QFS nature of the one-proton and one-neutron removal reactions can be validated by angular correlations of the recoiled target proton and the knocked out nucleon, which scatter at 
large angles. It was observed previously [25] that these reactions are close to coplanarity and that the particles have an opening angle of around $80-85^{\circ}$, due to the relativistic energies and to the non-zero binding energy of the nucleon in the nucleus.

In order to deduce the spectroscopic information of the single-particle states in question, a proper description by a theory is necessary as well. Particularly, the comparison of the experimental and theoretical cross-sections provides the reduction of the SP strength in form of spectroscopic or reduction factors. Additionally, from the momentum conservation in the Impulse Approximation (IA), the recoil momentum of the projectile-like reaction fragment can be linked to the internal momentum of the knocked out nucleon and hence, the orbital quantum number of the state from which it was knocked out [26]. The theory for ( $p, p n)$ and $(p, 2 p)$ reactions has recently been developed based on Glauber type calculations of multiple scattering, using the eikonal approximation [27]. The combined analysis of the experimental data and the theoretical calculations is still ongoing.

\section{Outlook}

Since the described experiment several upgrades and improvements have been made, especially to the detector system around the target area, which is very important for tracking, angular measurements and determination of final states of reaction fragments. Already now, the body of experimental data in hand indicates that the quasi-free knockout reactions off protons are a very useful tool for systematic studies of nuclear SP structure, including the stronger bound states. The experimental program at $\mathrm{R}^{3} \mathrm{~B}$ will highly benefit from the future FAIR facility, which will be able to deliver exotic nuclei with higher intensities.

\section{Acknowledgement}

The work was supported by the German Federal Ministry for Education and Research (BMBF project 05P12RDFN8), by HIC for FAIR and by GSI-TU Darmstadt Cooperation Contract.

\section{References}

[1] P.G. Hansen and J.A. Tostevin. Direct Reactions with Exotic Nulcei. Annu. Rev. Nucl. Part. Sci. 53 (2003) 219.

[2] L. Lapikás. Quasi-Elastic Electron Scattering off Nuclei. NPA 553 (1993) 297c.

[3] I. Sick. Correlations in nuclei. PPNP 59 (2007) 447.

[4] W.H. Dickhoff. Determining and calculating spectroscopic factors from stable nuclei to drip lines. J. Phys. G: Nucl. Part. Phys. 37 (2010) 064007.

[5] V.R. Pandharipande, I. Sick and P.K.A. deWitt Huberts. Independent particle motion and correlations in fermion systems. Rev. Mod. Phys. 69 (1997) 981.

[6] K. Sh. Egiyan, N. Dashyan, M. Sargsyan, S. Stepanyan L.B. Weinstein and SLAC collaboration. Observation of nuclear scaling in the A(e,e') reaction at $x_{B}$ 1. PRC 68 (2003) 014313.

[7] D. Rohe, et al. Correlated Strength in the Nuclear Spectral Function. PRL 93 (2004) 182501. 
[8] E. Piasetzky, et al. Evidence for Strong Dominance of Proton-Neutron Correlations in Nuclei. PRL 97 (2006) 162504.

[9] A. Gade, et al. Reduction of spectroscopic strength:Weakly-bound and strongly-bound single-particle states studied using one-nucleon knokcout reactions. PRC 77 (2008) 044306.

[10] C. Barbieri and W.H. Dickhoff. Spectroscopic factors in ${ }^{16} \mathrm{O}$ and Nucleon Asymmetry. Int. J. Mod. Phys. A 20 (2013) 1.

[11] R.J. Charity, L.G. Sobotka and W.H. Dickhoff. Asymmetry Dependence of Proton Correlations. PRL 97 (2006) 162503.

[12] T. Aumann. Neutron-rich Nuclei at and Beyond the Dripline in the Range $Z=4$ to $Z=10$ Studied in Kinematically Complete Measurements of Direct Reactions at Relativistic Energies. The R3B Collaboration, 2009.

[13] T. Aumann. Prospects of nuclear structure at the future GSI accelerators. PPNP 59 (2007) 3.

[14] G. Kraus, et al. Proton Inelastic Scattering on ${ }^{56}$ Ni in Inverse Kinematics. PRL 73 (1994) 1773.

[15] C.R. Gould, et al. Particle- $\gamma$-Ray Angular-Correlation Study of ${ }^{57} \mathrm{Ni}$ in the Reaction ${ }^{58} \mathrm{Ni}\left({ }^{3} \mathrm{He}\right.$, $\alpha \gamma)^{57} N i$. Phys. rev. 188 (1969) 1972.

[16] K.E. Rehm, et al. Study of the ${ }^{56} \mathrm{Ni}(d, p) 57 N i$ Reaction and the Astrophysical ${ }^{56} \mathrm{Ni}(p, \gamma) 57 \mathrm{Cu}$ Reaction Rate. PRL 80 (1998) 676.

[17] K.L. Yurkewicz, et al. One-neutron knockout from ${ }^{57}$ Ni. PRC 74 (2006) 024304.

[18] J. Lee, et al. Neutron spectroscopic factors of Ni isotopes from transfer reactions. PRC 69 (2009) 054611 .

[19] C. Barbieri. Role of Long-Range Correlations in the Quenching of the Spectrsocopic Factors. PRL 103 (2009) 202502.

[20] A. Gade, et al. Reduced Occupancy of the Deeply Bound Od $5 / 2$ neutron State om ${ }^{32}$ Ar. PRL 93 (2004) 042501 .

[21] J. Lee, et al. Neutron Spectroscopic factors of ${ }^{34}$ Ar and ${ }^{46}$ Ar from $(p, d)$ transfer reactions. PRC 83 (2011) 014606.

[22] O. Ershova. Coulomb Dissociation Reactions on Molybdenum Isotopes for Astrophysics Applications. $\mathrm{PhD}$ thesis, Johann Wolfgang Goethe-Universität, Frankfurt am Main (Germany), 2011.

[23] H. Geissel, et al. The GSI projectile fragment separator (FRS): a versatile magnetic system for relativistic heavy ions. Nucl. Instr. Meth. Phys. Research B70 (1992) 286.

[24] Th. Blaich, et al. A large area detector for high-energy neutrons: LAND collaboration. Nucl. Instr. Meth. Phys. Research A314 (1992) 136.

[25] S. Paschalis. Relativistic One-Nucleon Removal Reactions. PhD thesis, University of Liverpool (UK), 2008.

[26] H. Simon, et al. Systematic investigation of the drip-line nuclei ${ }^{11}$ Li and ${ }^{14} \mathrm{Be}$ and their unbound subsystems ${ }^{10} \mathrm{Li}$ and ${ }^{13} \mathrm{Be}$. NPA 791 (2007) 267.

[27] T. Aumann, C. Bertulani and J. Ryckebusch. Quasi-free ( $p, 2 p)$ and ( $p, p n)$ reactions with unstable nuclei. PRC 88 (2013) 064610. 